\title{
Multicenter urethroplasty outcomes for urethral stricture disease for patients with neurogenic bladder or bladder dysfunction requiring clean intermittent catheterization
}

\author{
Andrew J. Cohen ${ }^{1}$, Philip J. Cheng ${ }^{2}$, Sikai Song ${ }^{3}$, German Patino, ${ }^{3,4}$ Jeremy B. Myers ${ }^{2}$, Samit S. Roy ${ }^{5}$, \\ Sean P. Elliott ${ }^{5}$, Joseph Pariser ${ }^{5}$, Justin Drobish ${ }^{6}$, Brad A. Erickson ${ }^{6}$, Thomas W. Fuller ${ }^{7}$, Jill C. Buckley ${ }^{7}$, \\ Alex J. Vanni ${ }^{8}$, Nima Baradaran', Benjamin N. Breyer ${ }^{3}$ \\ ${ }^{1}$ James Buchanan Brady Urological Institute, Johns Hopkins Hospital, Baltimore, MD, USA; ${ }^{2}$ Department of Urology, University of Utah, Salt \\ Lake City, UT, USA; ${ }^{3}$ Department of Urology, Zuckerberg San Francisco General Hospital, University of California-San Francisco, San Francisco, \\ CA, USA; ${ }^{4}$ Hospital San Ignacio, Bogota, Colombia; ${ }^{5}$ University of Minnesota, Minneapolis, MN, USA; ${ }^{6}$ University of Iowa, Iowa City, IA, USA; \\ ${ }^{7}$ University of California, San Diego, CA, USA; ${ }^{8}$ Lahey Hospital and Medical Center, Burlington, MA, USA; ${ }^{9}$ Department of Urology, Ohio State, \\ Columbus, OH, USA \\ Contributions: (I) Conception and design: AJ Cohen, BN Breyer; (II) Administrative support: AJ Cohen, BN Breyer; (III) Provision of study materials \\ or patients: JB Myers, SP Elliot, BA Erickson, JC Buckley, AJ Vanni, BN Breyer; (IV) Collection and assembly of data: JB Myers, SP Elliot, BA \\ Erickson, JC Buckley, AJ Vanni, BN Breyer; (V) Data analysis and interpretation: All authors; (VI) Manuscript writing: All authors; (VII) Final \\ approval of manuscript: All authors. \\ Correspondence to: Benjamin N. Breyer, MD, MAS, FACS. Associate Professor of Urology, University of California, San Francisco, Zuckerberg San \\ Francisco General Hospital and Trauma Center, 1001 Potrero Suite 3A, San Francisco, CA 94110, USA. Email: Benjamin.Breyer@ucsf.edu.
}

Background: Our objective is to better comprehend treatment considerations for urethral stricture disease (USD) in patients requiring long-term clean intermittent catheterization (CIC). Patient characteristics, surgical outcomes and complications are unknown in this population.

Methods: Six members of the Trauma and Urologic Reconstruction Network of Surgeons (TURNS) participated in a prospective (2009 to present) and retrospective (prior to 2009) database recording patient demographics, surgical approach and outcomes. We included all patients undergoing urethroplasty who perform CIC. Descriptive statistics were used to analyze results.

Results: A total of 37 patients with 39 strictures were included. Bladder dysfunction was characterized as detrusor failure in 35\% and neurogenic etiology in 65\%. Median stricture length was $3 \mathrm{~cm}$ (IQR: 1.5-5.5) with $28 \%$ repaired with dorsal onlay buccal mucosal graft, $26 \%$ excision and primary anastomosis, $8 \%$ dorsal inlay, $8 \%$ ventral and dorsal, $8 \%$ flap based $8 \%$ non-transecting and $15 \%$ other. Functional success was 90\%: 4 patients required DVIU or dilation due to recurrence, with 2 of those ultimately requiring repeat urethroplasty. $86 \%$ of patients returned to CIC; no patients reported new pad use for urinary leakage after urethroplasty. During a median follow-up period of 3.1 years (IQR: 1.0-5.3), no patients underwent urinary diversion.

Conclusions: Urethroplasty is suitable, safe and effective for patients dependent on CIC suffering from USD. The effect of continual CIC on long-term outcomes remains uncertain.

Keywords: Spinal cord injuries; urethral stricture; urethroplasty; neurogenic bladder; urinary catheterization

Submitted Jun 09, 2020. Accepted for publication Mar 17, 2021.

doi: $10.21037 /$ tau-20-988

View this article at: http://dx.doi.org/10.21037/tau-20-988 


\section{Introduction}

A functional outlet is pivotal for any patient with neurogenic bladder or severe myogenic failure to allow for safe bladder drainage. Neurogenic bladder results from spinal cord injury, neurologic disease such as multiple sclerosis, or severe diabetic nephropathy (1). Patients with neurogenic bladder face increased risk of stone development, infection and renal dysfunction (1). Similar sequelae may result from myogenic failure and an acontractile detrusor (2). To regularly drain the bladder and protect against renal deterioration, clean intermittent catheterization (CIC) is a mainstay of bladder management in patients with neurogenic bladder or detrusor failure (3).

Unfortunately, urethral injury can result from improper, difficult, or traumatic, repeated catheterization (4). In patients requiring long-term CIC, particularly in those with impaired dexterity, the risk of urethral injury may be higher than that in the general population $(5,6)$. Given increased regular instrumentation, such patients face the possibility of urethral erosion, fistula, and urethral stricture disease (USD) (7). In particular for those patients with significant detrusor sphincter dyssynergia, the urethra could be traumatized when using force to pass through a spastic external sphincter. When USD occurs concomitant with CIC, many patients can be managed endoscopically: once the USD is opened by urethrotomy or dilation, continued CIC multiple times per day may help keep the stricture open.

Recalcitrant urethral strictures, or concurrent urethral false passages may render endoscopic management impossible in select men. When endoscopic management fails, management options include urinary diversion, catheterizable channel, permanent suprapubic tube (SPT) or urethroplasty $(1,5,8,9)$. Regarding urethroplasty in this context, the definition of success is unique and must consider how the reconstruction affects CIC. For instance, an open lumen with a sacculation may negatively affect catheter passage and urethral rest may especially impact surgical success. Moreover, the ideal management strategy for transitioning a patient back to CIC after urethroplasty is unknown. Even though there are unique considerations for urethroplasty in men on CIC, existing outcomes data are limited to small series consisting of $6-7$ patients each $(7,10)$. This paucity of data leads to severe limitations in preoperative counseling for patients selecting between bladder management options. Hence, we present our experience of urethral reconstruction for patients with USD in presence of neurogenic bladder or detrusor failure requiring CIC.
We present the following article in accordance with the STROBE reporting checklist (available at http://dx.doi. org/10.21037/tau-20-988).

\section{Methods}

\section{Study population}

Eligibility for this study included patients with neurogenic bladder on CIC or non-neurogenic bladder patients who CIC secondary to detrusor failure or other unknown cause. Patients who were doing self-dilation for stricture disease with a catheter or those solely with meatal pathology or bladder neck stenosis were excluded. We examined patients meeting criteria who underwent urethral reconstruction by six members of the Trauma and Urologic Reconstruction Network of Surgeons (TURNS). TURNS is a multiinstitutional group of specialty-trained urologists with focus in reconstructive urology, many are also involved in the clinical care and research of neurogenic bladder. We reviewed the surgical records from a prospective (years 2009 to 2019) and retrospective (2006 to 2009) database for patient demographics, surgical approach, and outcomes. No differences in data collection or protocol existed between groups. We also noted the typical perioperative management strategy for bladder drainage and return to CIC by the participating authors.

This study was conducted in accordance with the Declaration of Helsinki (as revised in 2013). The study was approved by the Institutional Review Board at each participating site (UCSF NO.: 14-13650) and informed consent was taken from all the patients.

\section{Variables of interest}

We collected data on stricture length, location (bulbar, penile, membranous, panurethra), surgical approach (dorsal onlay, ventral only, dorsal inlay, augmented dorsal onlay, lateral onlay, multi-stage repairs, and perineal urethrostomy), and complications. Complications were characterized using Clavien Grade (11). The TURNS urethroplasty follow up protocol involves a 3 month and 12 month cystoscopy (12). We defined anatomic success as the ability to atraumatically advance a $17 \mathrm{Fr}$ cystoscope beyond the area where the urethroplasty took place at most recent cystoscopic followup. Functional recurrence was defined as any patient who underwent repeat surgical treatment for recurrent stricture at the site of the original 
Table 1 Demographics of patients with neurogenic bladder or bladder dysfunction undergoing urethroplasty $(n=37)$

\begin{tabular}{|c|c|}
\hline Characteristic & $\begin{array}{l}\text { Median [interquartile } \\
\text { range] or } \mathrm{N}[\%]\end{array}$ \\
\hline Age & 52 [39-67] \\
\hline BMI & 27 [24-30] \\
\hline Follow up (years) & $3.1[1.0-5.3]$ \\
\hline \multicolumn{2}{|l|}{ Comorbidity } \\
\hline Hypertension & $10[27]$ \\
\hline Diabetes mellitus & 7 [19] \\
\hline Hyperlipidemia & $6[16]$ \\
\hline Coronary artery disease & $5[14]$ \\
\hline On anticoagulation & $4[11]$ \\
\hline Cancer & $3[8]$ \\
\hline Chronic obstructive pulmonary disease & $3[8]$ \\
\hline Immuno-suppressed & $2[5]$ \\
\hline \multicolumn{2}{|l|}{ Smoking history } \\
\hline Current & $6[16]$ \\
\hline Former & $15[41]$ \\
\hline Never & 16 [43] \\
\hline \multicolumn{2}{|l|}{ Reason for $\mathrm{CIC}$} \\
\hline Spinal cord injury & $14[38]$ \\
\hline Detrusor failure & $13[35]$ \\
\hline Spina bifida & $3[8]$ \\
\hline Diabetes mellitus & $2[5]$ \\
\hline Multiple sclerosis & $1[3]$ \\
\hline Cerebral palsy & $1[3]$ \\
\hline Trauma & $1[3]$ \\
\hline Childhood disease & $1[3]$ \\
\hline Spinal malignancy & $1[3]$ \\
\hline Non-ambulatory & $14[38]$ \\
\hline Use of upper extremities & $36[96]$ \\
\hline \multicolumn{2}{|l|}{ Bladder management strategy } \\
\hline Pre-stricture use of $\mathrm{CIC}$ & $32[86]$ \\
\hline Pre-stricture use of SPT & $3[8]$ \\
\hline Condom catheter use & $3[8]$ \\
\hline Temporary SPT (Urethral Rest) & $15[41]$ \\
\hline
\end{tabular}

SPT, suprapubic tube; CIC, clean intermittent catheterization; NGB, neurogenic bladder; BMI, body mass index. reconstruction during the follow up period, including: urethral dilation, direct vision internal urethrotomy, repeat urethroplasty, creation of catheterizable channel, or urinary diversion.

We performed further chart review to ascertain the reasons for bladder dysfunction and determine the patients' bladder management strategies. We also captured history germane to patients with spinal cord injury such as concomitant pressure ulcers and ambulatory status. We sought details of differences in pre-operative and postoperative bladder management.

\section{Statistical analysis}

Pertinent clinical details of the cohort were described with descriptive statistics as appropriate. Medians and interquartile ranges were reported. Stata 15 (College Station, Texas) and Excel (Redmond, California) was used for the analysis.

\section{Results}

\section{Study population}

Out of 2,661 anterior urethroplasties in the database we identified a cohort of 37 male patients who met inclusion criteria. They had with varied comorbid conditions with a median age of 52 years [interquartile range (IQR): 39-67]. Patient characteristics are described in detail in Table 1. A total of $23(62 \%)$ patients were ambulatory with $4(10.8 \%)$ reporting pressure ulcers. The median follow-up period was 3.1 years (IQR: 1.0-5.3). Bladder dysfunction was characterized as detrusor failure in $35 \%$, the remaining $65 \%$ had neurogenic bladder, with the etiology being spinal cord injury or spina bifida in the majority. Thirty-seven patients had one stricture, while two patients had strictures at separate time periods in non-overlapping areas within the urethra; hence we describe 39 strictures. CIC itself was the attributed cause of stricture development for at least $44 \%$ of patients, while the etiology in another $41 \%$ was idiopathic. CIC as a cause was noted when patients reported a recollection of an episode of difficult catheterization that resulted in trauma. 20/39 (51\%) strictures had prior treatment with urethral dilation or direct vision internal urethrotomy (DVIU). Such patients underwent a median of 1 [IQR: 1-3] endoscopic treatment prior to urethroplasty. One patient notably underwent over 30 prior endoscopic procedures for a prostatic/membranous urethral stricture. 
Table 2 Study population surgical and stricture details ${ }^{\ddagger}$

\begin{tabular}{|c|c|}
\hline Characteristic & $\begin{array}{c}\mathrm{N}[\%] \text { or median } \\
\text { (interquartile range) }\end{array}$ \\
\hline \multicolumn{2}{|l|}{ Etiology of stricture } \\
\hline Directly related to $\mathrm{CIC}$ & $17[44]$ \\
\hline Idiopathic & $16[41]$ \\
\hline Traumatic & $2[5]$ \\
\hline Other & $4[10]$ \\
\hline \multicolumn{2}{|l|}{ Location } \\
\hline Bulbar & $17[44]$ \\
\hline Penile & $11[28]$ \\
\hline Membranous & $5[13]$ \\
\hline Pan urethral & $6[15]$ \\
\hline \multicolumn{2}{|l|}{ Repair type } \\
\hline Dorsal onlay & $11[28]$ \\
\hline Anastomotic & $10[26]$ \\
\hline Dorsal inlay buccal & $3[8]$ \\
\hline Ventral \& dorsal & $3[8]$ \\
\hline Flap based & $3[8]$ \\
\hline Non-transecting & $3[8]$ \\
\hline Perineal urethrostomy & $2[5]$ \\
\hline Other & $4[10]$ \\
\hline \multicolumn{2}{|l|}{ Graft/flap type } \\
\hline Buccal & $18[46]^{\alpha}$ \\
\hline Fasciocutaneous & $3[8]$ \\
\hline None/Not specified & $18[46]$ \\
\hline Concurrent urethrocutaneous fistula & $1(2.7)$ \\
\hline Concurrent urethral erosion & $2(5.4)$ \\
\hline Length of stricture, $\mathrm{cm}$ & $3(1.5-5.5)$ \\
\hline Maximum graft length, $\mathrm{cm}$ & $6(3.5-7.5)$ \\
\hline
\end{tabular}

${ }^{a} 2$ buccal grafts used for 2 cases when simultaneous ventral and dorsal grafts were utilized; ${ }^{\ddagger} 39$ distinct strictures corrected in 37 patients, CIC, clean intermittent catheterization; other, meatoplasty, combination repairs, or unspecified.

The balance of patients, after shared decision making with their physicians, elected not to pursue endoscopic management with the severity of their disease in mind.

Urethral rest with SPT was pursued in 15 (41\%) patients for a median of 2 months (IQR: $1.25-5$ ) prior to
Table 3 Operative and functional outcomes

\begin{tabular}{lc}
\hline Characteristic & $\mathrm{N}[\%]$ \\
\hline Return to CIC & $32[86]$ \\
Voiding via urethra & $5[14]$ \\
Using SPT & $1[3]$ \\
Anatomic recurrence (by cystoscopy) & $8[22]$ \\
Functional recurrence (by secondary procedure) & $4[10]$ \\
Repeat DVIU & $4[10]$ \\
Repeat urethroplasty* & $2[5]$ \\
New pad use after urethroplasty & $0[0]$ \\
Daytime incontinence & 5 (13.5) \\
Nighttime incontinence & 5 (13.5) \\
\hline *2/2 underwent failed endoscopic management first. DVIU, \\
direct vision internal urethrotomy; SPT, suprapubic tube; CIC, \\
clean intermittent catheterization.
\end{tabular}

urethroplasty. Among included surgeons, 4 of 6 regularly retain SPT tubes post-operatively for up to 2 months to avoid CIC across a fresh urethroplasty whereas $2 / 6$ restart CIC after acceptable post-operative RUG. Patients presented with a median stricture length of $3 \mathrm{~cm}$ (IQR: 1.5-5.5) with diverse approaches to surgical repair (Table 2). There were no differences between stricture length for those undergoing pre-operative SPT vs. not $\mathrm{P}=0.16$. Dorsal onlay buccal mucosal graft repairs and excision and primary anastomosis accounted for $28 \%$ and $26 \%$ of repair types, respectively. Grafts were on average $6 \mathrm{~cm}$ long (IQR: $3.5-7.5 \mathrm{~cm}$ ).

\section{Outcomes}

Ultimately, 4 patients required repeated surgical intervention, which equates to a $90 \%$ functional success rate. $32(86 \%)$ patients returned to CIC for primary bladder management (Table 3), 5 (14\%) are spontaneous voiders, 4 by Credé and 1 by condom catheter and 1 (3\%) continues to use indwelling SPT by preference. The patient's typical post-surgical CIC catheter size was $14 \mathrm{Fr}$ (IQR: 12-14). Specific outcomes for patients with the longest strictures $(10,15$ and $16 \mathrm{~cm})$ were a return to CIC per urethra $(\mathrm{n}=1)$ and perineal urethrostomy with CIC $(n=2)$ with 1 patient requiring dilation of their urethrostomy. Eight patients had an anatomic recurrence on cystoscopy, but no hindrance of CIC. The initial surgery type (and stricture lengths) for those requiring re-intervention were excision and primary 
Table 4 Detailed surgical complications

\begin{tabular}{llc}
\hline Grade & Event & $\mathrm{N}[\%]$ \\
\hline Grade I & & $2[5]$ \\
& Scrotal Abscess & $1[3]$ \\
& Paraphimosis & $1[3]$ \\
Grade II & - & - \\
Grade III a & - & - \\
Grade III b & & $4[10]$ \\
& Balloon Dilation followed by EPA & $1[3]$ \\
& DVIU followed by urethroplasty & $1[3]$ \\
& Balloon dilation & $1[3]$ \\
& DVIU & $1[3]$ \\
Grade IVNV & - & - \\
\hline
\end{tabular}

EPA, excision and primary anastomosis; DVIU, direct vision internal urethrotomy.

anastamosis $(2.5$ and $1 \mathrm{~cm})$, non-transecting urethroplasty $(3 \mathrm{~cm})$, and perineal urethrostomy $(15 \mathrm{~cm})$. No patients undergoing urethroplasty with graft placements required subsequent reoperation. Urethral rest did not impact outcomes.

Among the $5(14 \%)$ patients noting incontinence posturethroplasty, 2 (40\%) experienced this de novo after surgery. Neither of these patients found the symptoms severe enough to regularly use pads. For 6 (16\%) patients, incontinence improved after urethroplasty. No patients in this cohort underwent urinary diversion. Complications occurred in $6(16 \%)$ patients (Table 4). Grade I complications included post-operative scrotal abscess and paraphimosis. The 4 patients that required repeated surgery for recurrent stricture were classified as long-term grade III b complications.

\section{Discussion}

Our results show that urethroplasty can be successful during medium-term follow-up in patients requiring CIC. The literature lacks data regarding urethral reconstruction in this patient population and this paper represents the largest multicenter study to date. Our functional success rate of $90 \%$ is comparable to those previously described in the non-neurogenic population, which ranges from $75-93 \%$ (12-15). Historically, it was believed that the success rate for urethroplasty in this patient population was lower, and thus, some urologists believed these patients may be better candidates for urinary diversion (10). It should be noted that most of our population was ambulatory and nearly all had functional upper extremities. Given the inherent complexity of the CIC patient population, shared decision making regarding early urinary diversion versus urethroplasty should likely involve high-volume, expert reconstructive surgeons (14). Our study suggests for well selected patients, urinary diversion can often be delayed or avoided with urethroplasty. No patient in this cohort required urinary diversion at a median follow up of 3.1 years.

The effect of CIC on recurrence after anastomotic or substitution urethroplasty rates remain unknown. There is some data evaluating USD recurrence after endoscopic management combined with self-dilation. Such studies have shown that DVIU followed by self-catheterization reduces USD recurrence by approximately half and longer duration of CIC after DVIU may lead to further risk reduction $(16,17)$. Notably, the urethral strictures in our study are considerably longer than those typically treated with DVIU $(17,18)$. Self-dilation after DVIU is often performed daily, every other day, or even weekly. For patients dependent on CIC, catheterization occurs 3-8 $\times$ per day to empty the bladder and may require irrigation of fluid or other maneuvers which lead to more contact time or microtrauma to the urethra.

Additionally, $44 \%$ of patients in this cohort developed strictures as a direct result of (self-reported) catheter trauma, a known risk factor for USD (5). In one study, Perrouin-Verbe et al. found that $19 \%$ of male patients doing CIC for a median of 9 years developed USD (19). Cornejo-Dávila recently described $4.2 \%$ of 250 men with neurogenic bladder developed a $14 \mathrm{Fr}$ stricture or worse over a mean follow-up of 19.8 months after commencing CIC (6). Attempts to improve the safety of CIC include use of hydrophilic catheters, patient education in technique, and continued evaluation of patient dexterity $(20,21)$. The data surrounding CIC is paradoxical as CIC may be used to decrease USD recurrence after DVIU but conversely may increase the risk of USD in the context of CIC for neurogenic bladder.

In our cohort, $41 \%$ of patients underwent a preoperative SPT placement. The literature supports SPT in lieu of downsizing the CIC catheter when a formal repair is planned $(22,23)$. While continuing CIC may maintain luminal patency, repeated CIC attempts in the face of a developing stricture may lead to hematuria, patient discomfort, urethral trauma and creation of false passages (22). 
Furthermore, allowing the scar to mature improves staging of USD and may improve the ability to perform urethroplasty (23). Indeed, it has been shown that a period of 'urethral rest', via placement of an SPT, altered the planned operative repair for urethroplasty almost half the time amongst expert reconstructive surgeons (24). Nonetheless, for the $59 \%$ of patients herein that did not have urethral rest, outcomes were still excellent. SPT management post-operatively remains an open question. Some surgeons in our study left a SPT post-operatively up to 2 months to avoid any temporary difficulty with CIC due to new angle or edema which may resolve in time. In our series, no particular post-operative management strategy was associated with a decreased risk of failure. It has been postulated the use of grafts might incur sacculation rendering CIC problematic or prone to failure; in this cohort all cases of failure occurred in cases without grafts.

Development of a urethral stricture in a patient dependent on CIC may represent an unwelcome opportunity to reflect on the patient's long-term bladder management plan. Specifically for patients with neurogenic bladder after spinal cord injury, CIC may be associate with lower patient satisfaction as compared to other management strategies such as surgery (25). Continuous SPT drainage is one option, but it may lead to a significant reduction in bladder capacity and is associated with increased risk of urinary tract infections, urosepsis, hydronephrosis, urinary calculi, renal insufficiency, all cause hospitalizations, and even a higher incidence of pressure ulcers $(5,26,27)$. Nonetheless, temporary SPT placement may enable patients to consider an alternate bladder management strategy and ensure that resumption of CIC after urethroplasty matches their preferences and goals.

The well selected patients in this cohort were able to at least delay urinary diversion as no patient require diversion during median 3 year follow up. In prior series from the early 2000's, 11 (64\%) patients who presented with varied urethral pathology (stricture, erosion, and/or fistula) ultimately underwent urinary diversion at a mean time of 3.3 years (10). Given development of botulinum toxin and improved surveillance, rates of augmentation cystoplasty or other invasive reconstructive options are on the decline $(28,29)$. In well selected patients, our results support the use of urethroplasty prior to creation of a catheterizable channel like mitrofanoff, given reported revision rates up to $38 \%$ for channels (30). The literature also suggests that patients with neurogenic bladder may incur higher perioperative risk for abdominal surgery, particularly because these patients often have an altered sensorium, which may lead to delayed recognition of complications (31). Increasing confidence in urethroplasty outcomes in this population may allow patients to quickly return to CIC and avoid long-term consequences of a devastated outlet such as major abdominal surgery or chronic SPT.

\section{Limitations}

The study represents a heterogeneous group of patients with varied reasons for being dependent on CIC presenting to genitourinary reconstructive experts. This represents a retrospective analysis with inherent bias, small sample size, without protocol for analyzing pre and postoperative continence or patient reported outcomes. The results may not be generalizable to all patients who CIC and develop USD. Importantly, many patients with NGB undergo immediate urinary diversion, and such patients were not captured by this study. Diagnosis and chronic management of bladder dysfunction was typically done prior to referral for stricture management; thus, urodynamics were not readily available in these patients. Furthermore, performing urodynamics in patients with USD is technically challenging. For that reason we lack data on presence or absence of detrusor sphincter dyssynergia, ranges of bladder pressures, and formal definition of underactive $v s$. neurogenic bladder in these patients. We acknowledge it can be difficult to pre-operatively differentiate between a stricture secondary to CIC $v s$. an idiopathic etiology as this is based on patient report. Any errors of omission from chart review, if present, would be non-differential to our results. In the case of the 2 patients with non-overlapping strictures, we strongly suspect the second occurrence did not result from the first reconstruction given the disparent regions and time intervals involved, hence they were not excluded or counted as 'failures'. Future work would be strengthened by including more pertinent patient-reported outcome measures for this patient population. Currently, bladder specific quality of life measurement tools for patients with neurologic conditions are heterogeneous and lack attention to the symptomatology of concomitant urethral stricture disease $(25,32)$. Details on methods and timing of the return to CIC after urethroplasty were heterogeneous and poorly recorded in the medical record.

\section{Conclusions}

For patients who are dependent on CIC and develop USD, 
urethroplasty is suitable and safe. Urethroplasty allows men to continue urethral CIC with high rates of success and low morbidity in the medium term. It avoids or at least delays urinary diversion for the well selected patient.

\section{Acknowledgments}

Mr. Anthony Enriquez, Clinical Research Coordinator at UCSF, worked directly with the authors to provide administrative and technical support for manuscript submission.

Funding: Funding was provided by the Alafi Foundation (Grant no. 10001).

\section{Footnote}

Reporting Checklist: The authors have completed the STROBE reporting checklist. Available at http://dx.doi. org/10.21037/10.21037/tau-20-988

Data Sharing Statement: available at http://dx.doi. org/10.21037/10.21037/tau-20-988

Peer Review File: Available at http://dx.doi. org/10.21037/10.21037/tau-20-988

Conflicts of Interest: All authors have completed the ICMJE uniform disclosure form (available at http://dx.doi. org/10.21037/10.21037/tau-20-988). Dr. Elliott reports grants from Urotronic, outside the submitted work. Dr. Vanni reports personal fees from Orchestra Biomed, outside the submitted work. The other authors have no conflicts of interest to declare.

Ethical Statement: The authors are accountable for all aspects of the work in ensuring that questions related to the accuracy or integrity of any part of the work are appropriately investigated and resolved. This study was conducted in accordance with the Declaration of Helsinki (as revised in 2013). The study was approved by the Institutional Review Board at each participating site (UCSF NO.:14-13650) and informed consent was taken from all the patients.

Open Access Statement: This is an Open Access article distributed in accordance with the Creative Commons Attribution-NonCommercial-NoDerivs 4.0 International License (CC BY-NC-ND 4.0), which permits the non- commercial replication and distribution of the article with the strict proviso that no changes or edits are made and the original work is properly cited (including links to both the formal publication through the relevant DOI and the license). See: https://creativecommons.org/licenses/by-nc-nd/4.0/.

\section{References}

1. Dorsher PT, McIntosh PM. Neurogenic bladder. Adv Urol 2012;2012:816274.

2. Osman NI, Chapple CR, Abrams P, et al. Detrusor Underactivity and the Underactive Bladder: A New Clinical Entity? A Review of Current Terminology, Definitions, Epidemiology, Aetiology, and Diagnosis. Eur Urol 2014;65:389-98.

3. Jamison J, Maguire S, McCann J. Catheter policies for management of long term voiding problems in adults with neurogenic bladder disorders. Cochrane Database Syst Rev 2013;(11):CD004375.

4. Mundy AR, Andrich DE. Urethral strictures. BJU Int 2011;107:6-26.

5. Weld KJ, Dmochowski RR. Effect of bladder management on urological complications in spinal cord injured patients. J Urol 2000;163:768-72.

6. Cornejo-Dávila V, Durán-Ortiz S, Pacheco-Gahbler C. Incidence of Urethral Stricture in Patients With Spinal Cord Injury Treated With Clean Intermittent SelfCatheterization. Urology 2017;99:260-4.

7. Casey JT, Erickson BA, Navai N, et al. Urethral reconstruction in patients with neurogenic bladder dysfunction. J Urol 2008;180:197-200.

8. Colli J, Lloyd LK. Bladder neck closure and suprapubic catheter placement as definitive management of neurogenic bladder. J Spinal Cord Med 2011;34:273-7.

9. Feifer A, Corcos J. Contemporary role of suprapubic cystostomy in treatment of neuropathic bladder dysfunction in spinal cord injured patients. Neurourol Urodyn 2008;27:475-9.

10. Secrest CL, Madjar S, Sharma AK, et al. Urethral reconstruction in spinal cord injury patients. J Urol 2003;170:1217-1221; discussion 1221.

11. Clavien PA, Barkun J, de Oliveira ML, et al. The ClavienDindo classification of surgical complications: five-year experience. Ann Surg 2009;250:187-96.

12. Erickson BA, Elliott SP, Voelzke BB, et al. Multiinstitutional 1-Year Bulbar Urethroplasty Outcomes Using a Standardized Prospective Cystoscopic Follow-up Protocol. Urology 2014;84:213-6. 
13. Eltahawy EA, Virasoro R, Schlossberg SM, et al. Longterm followup for excision and primary anastomosis for anterior urethral strictures. J Urol 2007;177:1803-6.

14. Faris SF, Myers JB, Voelzke BB, et al. Assessment of the Male Urethral Reconstruction Learning Curve. Urology 2016;89:137-42.

15. Mangera A, Patterson JM, Chapple CR. A systematic review of graft augmentation urethroplasty techniques for the treatment of anterior urethral strictures. Eur Urol 2011;59:797-814.

16. Bødker A, Ostri P, Rye-Andersen J, et al. Treatment of recurrent urethral stricture by internal urethrotomy and intermittent self-catheterization: a controlled study of a new therapy. J Urol 1992;148:308-10.

17. Khan S, Khan RA, Ullah A, et al. Role of clean intermittent self catheterisation (CISC) in the prevention of recurrent urethral strictures after internal optical urethrotomy. J Ayub Med Coll Abbottabad 2011;23:22-5.

18. Wessells H, Angermeier KW, Elliott S, et al. Male Urethral Stricture: American Urological Association Guideline. J Urol 2017;197:182-90.

19. Perrouin-Verbe B, Labat JJ, Richard I, et al. Clean intermittent catheterisation from the acute period in spinal cord injury patients. Long term evaluation of urethral and genital tolerance. Paraplegia 1995;33:619-24.

20. Oh SJ, Ku JH, Lim SH, et al. Effect of a "centralized intensive education system" for clean intermittent selfcatheterization in patients with voiding dysfunction who start catheterization for the first time. Int J Urol 2006;13:905-9.

21. De Ridder DJ, Everaert K, Fernández LG, et al. Intermittent catheterisation with hydrophilic-coated catheters (SpeediCath) reduces the risk of clinical urinary tract infection in spinal cord injured patients: a prospective randomised parallel comparative trial. Eur Urol 2005;48:991-5.

22. Kashefi C, Messer K, Barden R, et al. Incidence and prevention of iatrogenic urethral injuries. J Urol 2008;179:2254-7; discussion 2257-8.

Cite this article as: Cohen AJ, Cheng PJ, Song S, Patino G, Myers JB, Roy SS, Elliott SP, Pariser J, Drobish J, Erickson BA, Fuller TW, Buckley JC, Vanni AJ, Baradaran N, Breyer BN. Multicenter urethroplasty outcomes for urethral stricture disease for patients with neurogenic bladder or bladder dysfunction requiring clean intermittent catheterization. Transl Androl Urol 2021;10(5):2035-2042. doi: 10.21037/tau-20-988
23. Terlecki RP, Steele MC, Valadez C, et al. Urethral rest: role and rationale in preparation for anterior urethroplasty. Urology 2011;77:1477-81.

24. Moncrief T, Gor R, Goldfarb RA, et al. Urethral Rest with Suprapubic Cystostomy for Obliterative or Nearly Obliterative Urethral Strictures: Urethrographic Changes and Implications for Management. J Urol 2018;199:1289-95.

25. Myers JB, Lenherr SM, Stoffel JT, et al. Patient reported bladder-related symptoms and quality of life after spinal cord injury with different bladder management strategies. J Urol 2019;202:574-84.

26. Cameron AP, Wallner LP, Forchheimer MB, et al. Medical and psychosocial complications associated with method of bladder management after traumatic spinal cord injury. Arch Phys Med Rehabil 2011;92:449-56.

27. Kennelly M, Thiruchelvam N, Averbeck MA, et al. Adult Neurogenic Lower Urinary Tract Dysfunction and Intermittent Catheterisation in a Community Setting: Risk Factors Model for Urinary Tract Infections. Adv Urol 2019;2019:2757862.

28. Schlomer BJ, Saperston K, Baskin L. National trends in augmentation cystoplasty in the 2000s and factors associated with patient outcomes. J Urol 2013;190:1352-7.

29. Biers SM, Venn SN, Greenwell TJ. The past, present and future of augmentation cystoplasty. BJU Int 2012;109:1280-93.

30. O'Connor EM, Foley C, Taylor C, et al. Appendix or Ileum - Which is the Best Material for Mitrofanoff Channel Formation in Adults? J Urol 2019;202:757-62.

31. Benjamin E, Haltmeier T, Karamanos E, et al. Paraplegic and quadriplegic patients undergoing emergency abdominal surgery: sicker presentations, worse outcomes. J Trauma Acute Care Surg 2015;78:808-815; quiz 893-894.

32. Patel DP, Elliott SP, Stoffel JT, et al. Patient reported outcomes measures in neurogenic bladder and bowel: A systematic review of the current literature: Patient Reported Outcomes in Neurogenic Bladder and Bowel. Neurourol Urodyn 2016;35:8-14. 